\title{
Implementation of Dynamic System Approach for Radio Location Fingerprinting in WLANs
}

\author{
K. Divya \\ P.G Student \\ Malla Reddy College of \\ Engineering \& technology
}

\author{
M. Anusha \\ Assistant Professor \\ Malla Reddy College of \\ Engineering \& Technology
}

\author{
S. Anusha \\ Assistant Professor \\ K.G.Reddy College of \\ Engineering \& Technology
}

\begin{abstract}
Wireless indoor positioning systems have become very popular in recent years. These systems have been successfully used in many applications such as asset tracking and inventory management. Three typical location estimation schemes of triangulation, scene analysis, and proximity are analyzed. And also location fingerprinting in detail since it is used in most current system or solutions. This project focuses on the localization using Received Signal Strength (RSS) in dense multipath indoor environments. A dynamic system approach is proposed in the fingerprinting module, where the location is estimated from the state instead from RSS directly. The state is reconstructed from a temporal sequence of RSS samples by incorporating a proper memory structure based on Taken's embedded theory. Then, a more accurate statelocation correlation is estimated because the impact of the temporal variation due to multipath is considered.
\end{abstract}

\section{General Terms}

Triangulation, scene analysis, proximity

\section{Keywords}

Dynamic system, RSS, location fingerprinting, IEEE 802.11, WLAN, multipath.

\section{INTRODUCTION}

In todays day to day life the emergency services, demand for knowledge of location has increased drasticalnly [1], [2]. Among different techniques a popular indoor positioning technique to date is a two-stage location fingerprinting [3], [4]. Recently, so many number of fingerprinting systems are developed based on Received Signal Strength (RSS) of the radio in Wireless Local Area Networks (WLANs) in order to avoid extra costs [5]-[8]. This design involves a database called radio map that stores pre-recorded RSS at reference positions. Then, the location is inferred by comparing a new RSS with the offline constructed radio fingerprints [5], [9]. However, the signal propagation in indoor environments is extremely complex and RSSs are usually varying with time even at a fixed location [10]. These temporal variations, incur a characteristic mismatch between the fingerprints and the online observation, and thus, result in various degrees of accuracy degradation [11]-[13].Many studies have addressed the issues of RSS variations. For example, the history of either estimates or fingerprints is utilized in probabilistic approaches to reduce its impact by averaging within a certain period [11], [14]. The radio map is temporally adapted to the current environment by in using sensors [15]. Some works utilized a power delay profile to represent the multipath [16]-[18].
Whatever, the delay may not directly reflect the multipath in WLANs because the operation is dependent on the user's behavior to avoid the collision.

The time-varying property of RSS is caused due to number of reasons such as the multipath, shadow fading effect and how the terminal is held [19], [20]. We propose a dynamic system approach into the fingerprinting module to exploit the characteristic of the multipath effect. The concept of a dynamic system has been exploited in many applications such as Kalman filter [21]. The former smoothes the measurements while the later multiplies the observed probability of each state. In contrast, our approach adopts Taken's embedded theory [23]. The output of our dynamic system not only depends on the input but also the state information of the system. The state can be reconstructed from a sequence of observations of the dynamic system. To model a dynamic system, the model needs to be equipped with the memory [22]. By incorporating memory architecture into the positioning system, the location information is then exploited in the projected embedded dimension which is constructed by the temporal trajectory of a sequence of RSS observations of multiple access points (APs).

In this approach, the novel in the sense that the impact of the temporal fluctuations due to multipath is taken into consideration. This is motivated by the physical properties of the multipath propagation, where a new observation is actually involving the past samples. In other words, the spatial correlation is estimated between a states in the embedded dimension reconstructed from a temporal trajectory instead from RSS directly. In our positioning system, the kernel-based function is used to exploit the correlation. The experiments, performed in a real WLAN environment, demonstrate the usefulness of our approach to significant performance improvements.

\section{MULTIPATH PROPAGATION IN DYNAMIC SYSTEM}

In general, the measured RSS in a multipath induced environment can be written as

$\mathrm{x}(t)=\sum_{T=0}^{N-1} \alpha(\tau) \cdot h(\tau) \cdot[s(t-\tau)] e^{j \phi(\tau)}+g(t), \quad$ where $\mathrm{x}(t)$ means

the RSS observation at time $t$ and $h(\tau)$ and $\phi(\tau)$, respectively, represent the amplitude and relative phase of the delayed multipath components. The total number of delayed paths is equal to $N-1 . \alpha(\tau)$ is a binary function that controls the on-off activity of the corresponding multipath filter $h(\tau), s(t)$ is the ideal signal in a free space and $\tau$ is the time delay. 


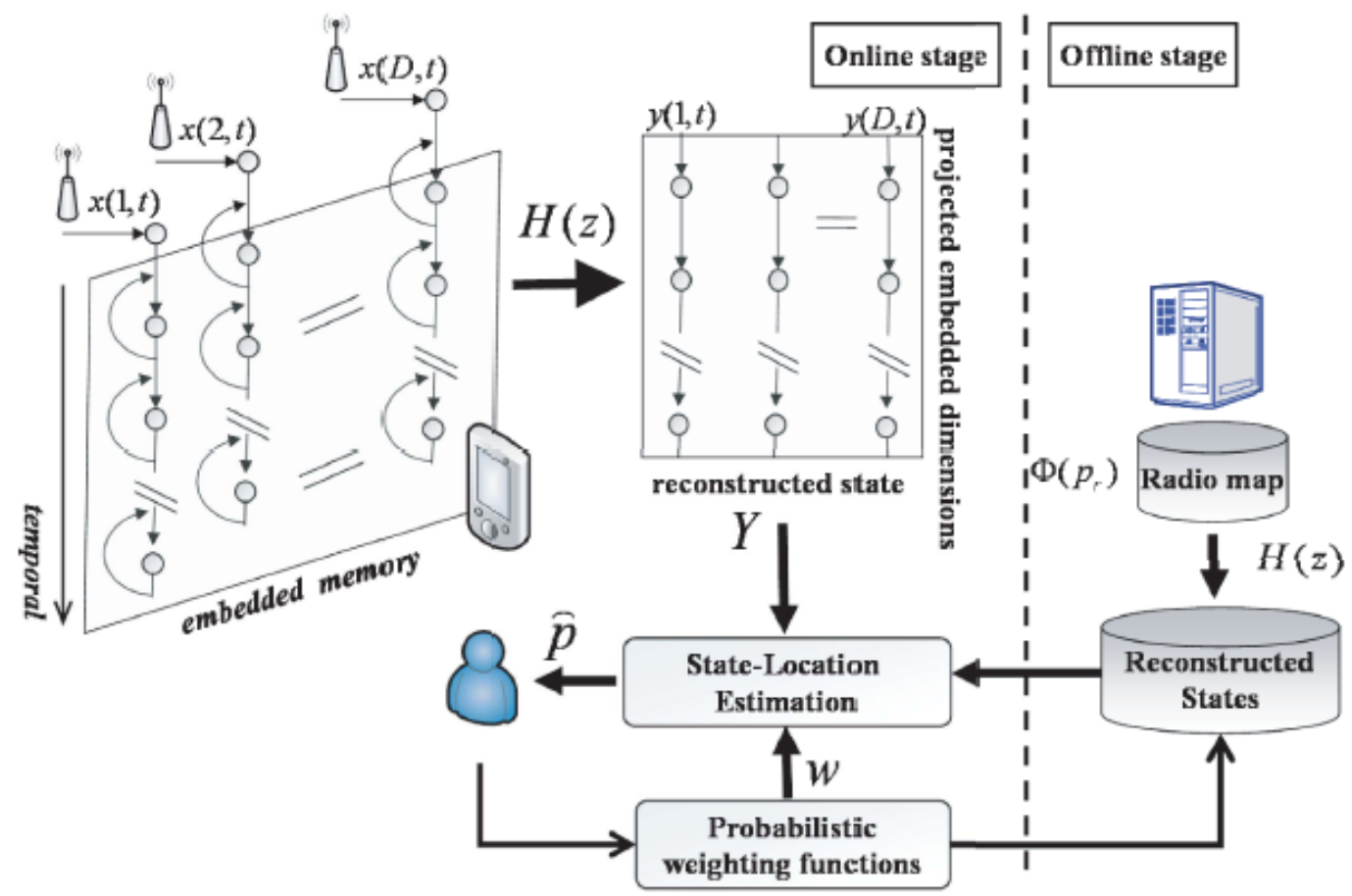

Figure 1.The system flow of dynamic approach utilizing the projected memory

$\mathrm{g}(t)$ is the communication noise, and in general, this noise contains everything not included in the summation term representing the multipath model. Clearly, the observation of RSS is in fact involving the previous samples with different weights. More precisely, each observation at time $t$ is the weighted linear combination of the past $s(t-\tau)$ and the corresponding multipath gain $H(\tau)=h(\tau) \alpha(\tau) e^{j \phi}(\tau)$. That is, the location information has strong relationship with respect to the temporal sequence of RSSs.

Most of current fingerprinting solutions are static in the sense that the location is estimated from only a single measurement as $\Re^{D} \rightarrow \Re^{2}$ [3]. Such a solution can be formulated as $\hat{p}(t)=$ $\mathbf{F}\{\mathbf{x}(t)\}$, where $\mathbf{x}(t) \in \mathfrak{R}^{D}$ is observed RSS from $D$ APs at time $t, \hat{p}(t) \in \mathfrak{R}^{2}$ represents the $2 D$ Cartesian coordinate of the estimated position and $\mathbf{F}$ is a RSS-position mapping function. Such characteristics motivates us that the location information should be extracted by first applying the inverse function $\mathrm{H}^{-1}(\tau)$. In this way, the positioning solution can be modified as $\hat{p}(t)=\mathbf{F}\left\{\mathbf{H}^{-\mathbf{1}}[\mathbf{x}(t)]\right\}$, where $\mathbf{H}^{-\mathbf{1}}$ represents the inverse multipath effect with multiple dimensions, it can be modeled as a finite impulse response (FIR) or an infinite impulse response (IIR) filter from the signal processing perspective [24].

After taking such characteristics into consideration, this paper presents a dynamic system approach incorporating probabilistic functions to location fingerprinting in WLAN . Dynamic systems are different from static systems in the sense that the former have memory. The output of a dynamic system not only depends on the input but also the state information of the system. Memory elements preserve the state information. In order to preserve the state information, we propose to introduce embedded memory [23], [25] into the fingerprinting module in order to model the dynamic characteristics of the multipath effect. According to Takens' embedded theorem [22], the state of a dynamic system can be reconstructed from a sequence of observations of the system. To model a dynamic system, the model needs to be equipped with the memory The simplest memory element is the unit time delay, which has the transfer function $H(Z)=Z^{-1}$. The simplest memory architecture is the tapped delay line, which consists of a series of $\mathrm{k}$ time delays. Resolution refers to the degree to which information concerning the individual elements of the input sequences is saved. A low-resolution memory holds coarse information about the input data. The depth of the memory refers to how far into the past the memory holds the input information. For the tapped delay line, the depth is the number of delay units and the resolution is 1 .

\section{ADVANCEPOSITING ALGORITHM FOR DYNAMIC FINGERPRINTING}

The advance positioning algorithm is based on the concept of dynamic systems and machine learning techniques. This approach differs from the traditional fingerprinting approaches in the following two aspects. First, the states are reconstructed from the projected embedded memory (temporal trajectory) of observation $x(t)$ by a transfer function $H(z)$. Second, the spatial correlation is estimated between the reconstructed states with the memory depth $N$. In other words, the system inputs are not only a current observation, but rather a reconstructed state. In this section, we describe how to construct the dynamic systems based on the probabilisticbased approaches.

The measured RSS $x(d, t)$ be defined for $t$ in the temporal dimension and for $d$ in the AP spatial domain. The inverse multipath $\mathbf{H}^{-1}$ can be viewed as a temporal filter1, the memory structure can be generally represented as the combination of filter input $\mathcal{X}(d, t)$ and the previous filter output. Let the memory depth is $N$, the filter output states $y(d, t)$ can be obtained by the following equation: 
$y(d, t)=\sum_{\tau=0}^{N-1} b_{d n} x(d, t-\tau)+\sum_{\tau=0}^{N-1} a_{d n} y(d, t-\tau)$

In this $\mathcal{y}(d, t)$ is the filter output at time $t$ and from the $d$ th AP, and $a_{d n}$ and $b_{d n}$ are, respectively, the filter coefficients weighting the previous outputs and inputs. For the notation purpose, let $\mathbf{Y}$ be the matrix form representing the reconstructions with $N$ memory depths and $D$ APs.

$\mathrm{Y}=\left(\begin{array}{ccc}y(1, t) & \mathrm{K} & y(1, t-N+1) \\ \mathrm{M} & \mathrm{O} & \mathrm{M} \\ y(D, t) & \mathrm{L} & y(D, t-N+1)\end{array}\right)_{D \times N}$

For probabilistic-based approaches, the positioning in a dynamic system can be formulated as follows:

$\hat{p}(t)=\sum_{r=1}^{R} \omega\left[Y, H\left(\phi\left(p_{r}\right)\right)\right] \cdot p_{\mathrm{r}}$

where $\mathbf{Y}$ is the reconstructed states from the online measurements, $\mathbf{p}_{\mathbf{r}} \in \mathfrak{R}^{2}$ is the coordinate of the $r$-th reference position and the value of $R$ is the number of available reference locations. The weighting function $w$ means the probability interpolating the $R$ reference positions, $\boldsymbol{\Phi}\left(p_{r}\right)$ represents the collected fingerprints at $\mathbf{p}_{\mathbf{r}}$, and $\mathbf{H}\left(\Phi\left(\mathbf{p}_{\mathbf{r}}\right)\right)$ means that all the fingerprints are passing through the same transfer function. $\mathbf{H}\left(\Phi\left(\mathbf{p}_{\mathbf{r}}\right)\right)=\left[\mathbf{y}_{r}(1) ; \cdots ; \mathbf{y}_{r}(L)\right]$ where $L$ means the number of collected fingerprints and $\mathbf{y}_{r} \in \mathfrak{R}^{D}$ is RSS from $D$ APs and at location $\mathbf{p}_{r}$. A matrix form of $\mathbf{H}\left(\Phi\left(\mathbf{p}_{\mathbf{r}}\right)\right)$ is defined as:

$$
H\left(\phi\left(p_{r}\right)\right)=\left(\begin{array}{ccc}
y_{r}(1,1) & \mathrm{K} & y_{r}(1, L) \\
\mathrm{M} & \mathrm{O} & \mathrm{M} \\
y_{r}(D, 1) & \mathrm{L} & y_{r}(D, L)
\end{array}\right)_{D \times L}
$$

Since the weighting function $\boldsymbol{\omega}$ is obtained between the state pattern $\mathbf{Y}$ and the fingerprints $\mathbf{H}\left(\Phi\left(\mathbf{p}_{\mathbf{r}}\right)\right)$, the proposed approach is dynamic. A special case of the dynamic approach occurs when $N=1$, which can be viewed as a static fingerprinting solution. That is, only the current observation is available for inputs of the estimation module.

The system flow of the proposed dynamic fingerprinting system is plotted in Fig. 1. During the offline stage, the states of the radio map are reconstructed by the same $H(Z)$ and the required parameters are prepared for location estimation. During the online stage, a sequence of consecutive samples are utilized to obtain the state information $\mathbf{Y}$. Then the state location correlation is estimated depending on the selected probabilistic approaches. The probabilistic approaches treats RSS at each location as a statistical random variable [14], [26]. In this article, the kernel-based methods are used here to exploit the probabilistic weighting $(\cdot)$ [27].

Before applying kernel-based techniques, the consecutive samples in the radio map are passed through the selected $H(Z)$ into a sequence of reconstructed states.

This way, $L-N$ state vectors with the same memory structure, denoted as $\mathbf{S}_{r}$, are generated for each location $\mathbf{p}_{r}$. Then, the kernel function can be performed on each state vector due to a dimension agreement as follows:

$\omega\left[Y, H\left(\phi\left(p_{r}\right)\right)\right]=\frac{1}{L-N} \sum_{i=1}^{L-N} \mathbf{K}(\mathbf{Y}, \mathbf{S}(i))$ where $\mathbf{K}$ is the kernel function calculating the nonlinear distance between $\mathbf{Y}$ and each $\mathbf{S}_{r}(i)$. Considering the normalization, the kernel function becomes:

$$
K\left(Y, S_{r}(i)\right)=\frac{\mathbf{K}\left(\mathbf{Y}, \mathbf{S}_{r}(i)\right)}{\sqrt{k(Y, Y) k\left(\mathbf{S}_{r}(i), \mathbf{S}_{r}(i)\right)}}
$$

The commonly used Gaussian kernel is adopted in our approach, we obtain

$$
K\left(Y, S_{r}(i)\right)=\frac{1}{L-N} \sum_{i=1}^{L-N} \exp \left(\frac{-\mathrm{PY}-\hat{\mathrm{S}}_{\mathrm{r}}(i) \mathrm{P}^{2}}{2 \sigma^{2}}\right)
$$

where $\sigma$ is an adjustable parameter and the operation $\|(\cdot)\|$ represents the norm function as:

$$
\mathrm{PY}_{-\hat{\mathrm{S}}_{r}}(i) \mathrm{P}^{2}=<\mathrm{Y}, \mathrm{S}_{\mathrm{r}}(i)>=\sum_{\tau=0}^{N-1} \sum_{d=1}^{D} y(d, t-\tau) y_{r}(d, \tau+1)
$$

where $<>$ indicates a typical inner product function.

\section{PERFORMANCE EVALUTION}

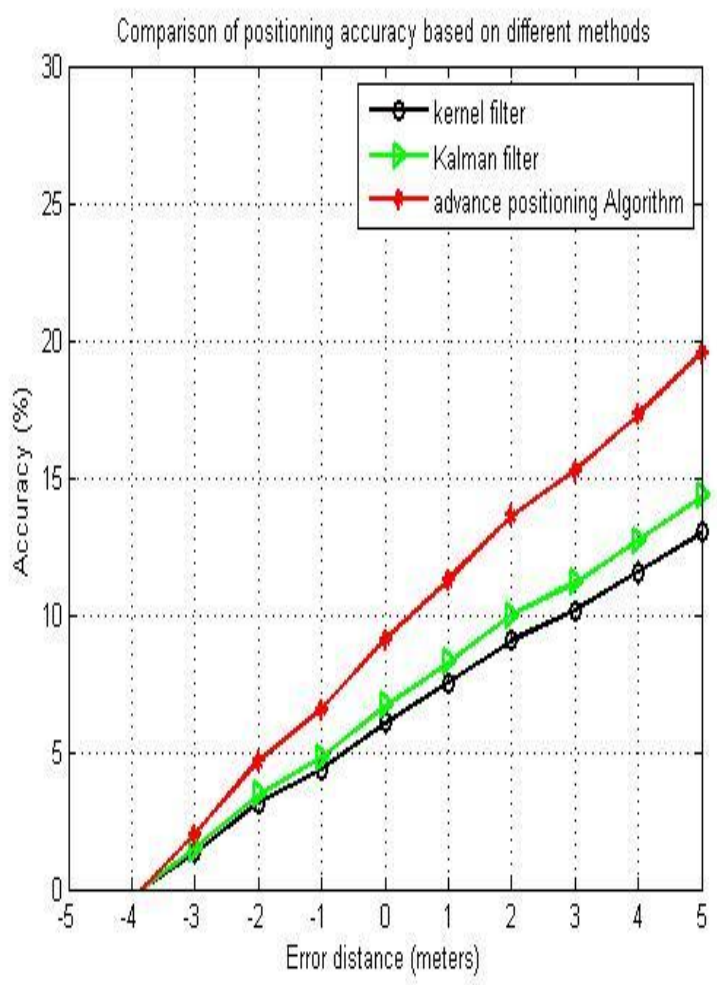

Figure2: Comparison of positioning accuracy based on different methods.

From figure 2 it is clearly that the state information is reconstructed to more accurately the spatial correlation in the proposed dynamic approach. By incorporating the memory architecture and estimating between a state, the performance is significantly enhanced since the temporal variation due to multipath is taken into consideration. That is why my approach achieves the better performance than the traditional approaches. 


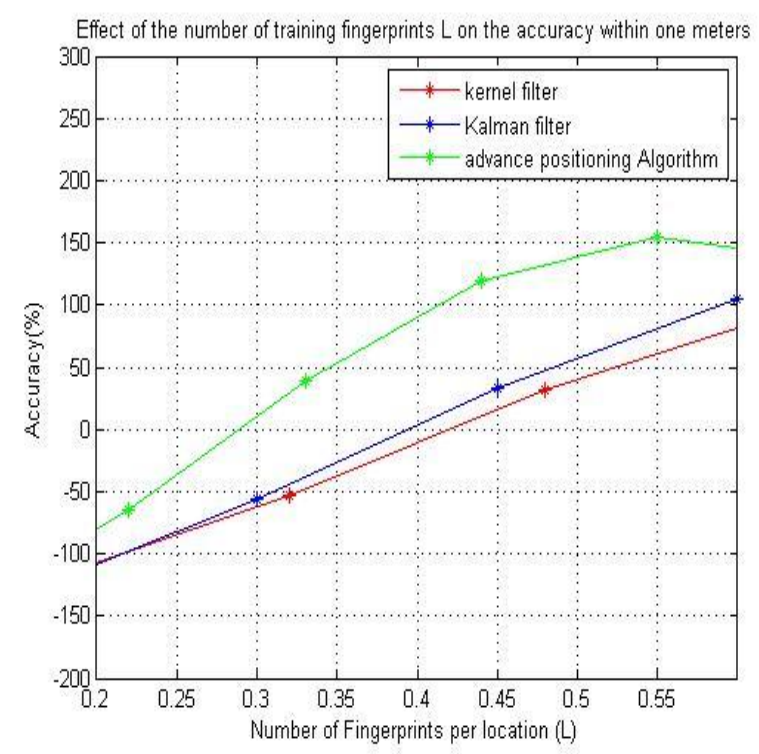

Figure 3: Effect of the number of training fingerprints $L$ on the accuracy within a short distance

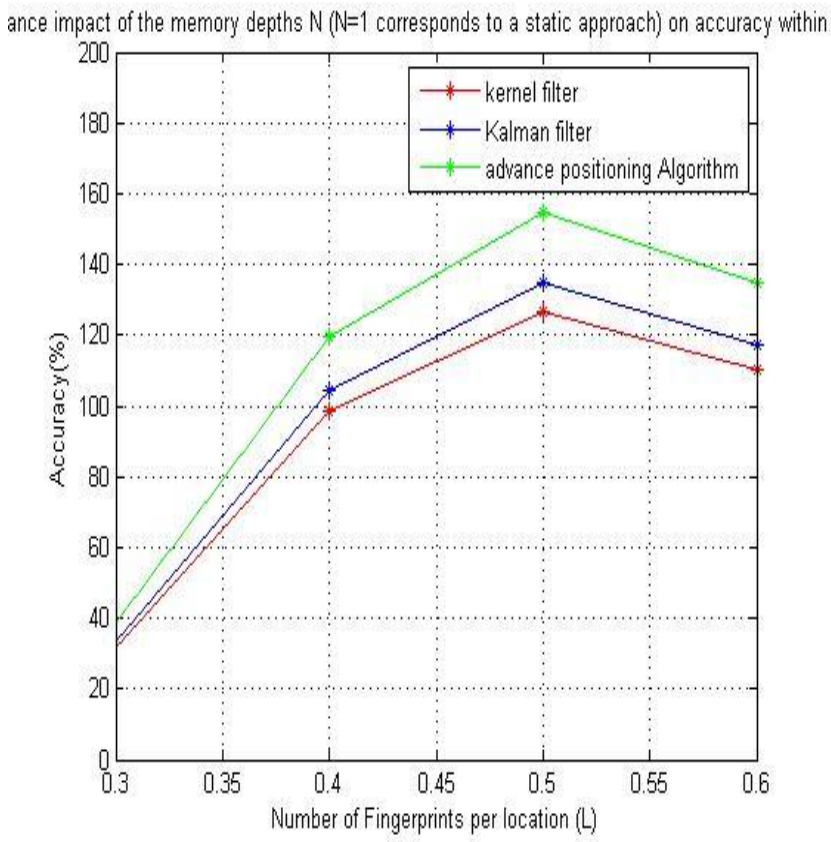

Figure 4: Performance impact of the memory depths $N$ on accuracy within a short distance.

The number of training fingerprints and the depths of memory. Figure 3 and Figure 4 shows the effect of different number of training fingerprints L per reference location. we can seen, the dynamic approach improves the accuracy with all values of $\mathrm{L}$. We learn that our approach has the advantage of using the fewest fingerprints to achieve the same accuracy.

\section{CONCLUSION}

The multipath propagation property inspires us that the location information is highly related to a temporal sequence of RSS samples. Thus, a novel dynamic system approach is developed into the WLAN fingerprinting module, where the output not only depends on current RSS, but also the state information of the system.
By incorporating a memory structure, the state information is reconstructed from a temporal sequence of RSS samples. The spatial correlation is then estimated from a state in the projected embedded dimension instead from RSS directly. In this method, we use the kernel-based function to exploit the correlation and compare with other state-based methods. The experiments are performed by collecting realistic RSS data in an indoor WLAN environment.

The results show that the positioning accuracy is significantly improved with the use of a dynamic system approach. The results support the conclusion that the temporal fluctuation due to multipath is taken into consideration in our algorithm.

\section{ACKNOWLEDGMENTS}

I would like to thank my guide who has supported me in all aspects and I would like to one of my sir who has suggested and helped me to do yhis project.

\section{REFERENCES}

[1] D. Munoz, F. Bouchereau, C. Vargas, and R. EnriquezCaldera, Position Location Techniques and Applications. Academic Press, 2009.

[2] S. Zafer, G. Sinan, and G. Ismail, Ultra-Wideband Positioning Systems: Theoretical Limits, Ranging Algorithms, and Protocols. Cambridge, 2008.

[3] M. Kjærgaard, "A taxonomy for radio location fingerprinting," Lecture Notes Computer Science, vol. 4718, pp. 139-156, 2007.

[4] S.-H. Fang, T.-N. Lin, and P.-C. Lin, "Location fingerprinting in a decorrelated space," IEEE Trans. Knowledge Data Eng., vol. 20, no. 5, pp. 685-691, 2008.

[5] V. Honkavirta, T. Perala, S. Ali-Loytty, and R. Piche, "A comparative survey of WLAN location fingerprinting methods," Positioning, Navigation Commun., pp. 243 251, 2009.

[6] J. J. Pan, J. T. Kwok, Q. Yang, and Y. Chen, "Multidimensional vector regression for accurate and low-cost location estimation in pervasive computing," IEEE Trans. Knowledge Data Eng., vol. 18, no. 9, pp. 1181-1193, 2006.

[7] S.-H. Fang and T.-N. Lin, "Indoor location system based on discriminant-adaptive neural network in IEEE 802.11 environments," IEEE Trans. Knowledge Data Eng., vol. 19, no. 11, pp. 1973-1978, 2008.

[8] Y. Chen, J. Yin, X. Chai, and Q. Yang, "Power-efficient access-point selection for indoor location estimation," IEEE Trans. Knowledge Data Eng., vol. 18, no. 7, pp. 877-888, 2006.

[9] X. Chai and Q. Yang, "Reducing the calibration effort for probabilistic indoor location estimation," IEEE Trans. Mobile Comput., vol. 6, no. 6, pp. 649-662, 2007.

[10] K. Kaemarungsi and P. Krishnamurthy, "Properties of indoor received signal strength for WLAN location fingerprinting," Mobile Ubiquitous Syst., Networking Services, pp. 14-23, 2004.

[11] M. Youssef and A. Agrawala, "Handling samples correlation in the Horus system," in Proc. IEEE INFOCOM, 2004, pp. 1023-1031.

[12] S.-P. Kuo and Y.-C. Tseng, "A scrambling method for fingerprint positioning based on temporal diversity and 
spatial dependency," IEEE Trans. Knowledge Data Eng., vol. 20, no. 5, pp. 678-684, 2008.

[13] S.-H. Fang, T.-N. Lin, and K.-C. Lee, "A novel algorithm for multipath fingerprinting in indoor WLAN environments," IEEE Trans. Wireless Commun., vol. 7, no. 9, pp. 3579-3588, 2008.

[14] T. Roos, P. Myllymaki, H. Tirri, P. Misikangas, and J. Sievanen, "A probabilistic approach to WLAN user location estimation," Wireless Inf. Networks, vol. 9, no. 3, pp. 155-164, 2002.

[15] J. Yin, Q. Yang, and L. M. Ni, "Learning adaptive temporal radio maps for signal-strength-based location estimation," IEEE Trans. Mobile Comput., vol. 7, pp. 869-883, July 2008.

[16] M. Triki, D. Slock, V. Rigal, and P. Francois, "Mobile terminal positioning via power delay profile fingerprinting: reproducible validation simulations," in Proc. IEEE Veh. Technol. Conf., 2006, pp. 1-5.

[17] C. Nerguizian, C. Despins, and S. Affes, "Geolocation in mines with an impulse response fingerprinting technique and neural networks," IEEE Trans. Wireless Commun., vol. 5, no. 3, pp. 603-611, 2006.

[18] Z. li Wu, C. hung Li, J.-Y. Ng, and K. R. Leung, "Location estimation via support vector regression," IEEE Trans. Mobile Comput., vol. 6, no. 3, pp. 311-321, 2007.

[19] T. Wigren, “Adaptive enhanced cell-ID fingerprinting localization by clustering of precise position measurements," IEEE Trans. Veh. Technol., vol. 56, no. 5, pp. 3199-3209, 2007.
[20] M. Simic and P. Pejovic, "An algorithm for determining mobile station location based on space segmentation," IEEE Commun. Lett., vol. 12, no. 7, pp. 499-501, 2008.

[21] F. F. Gene, L. W. Michael, and P. Dave, Digital Control of Dynamic Systems. Boston: Addison-Wesley Longman, 1997.

[22] F. Taken, "Detecting strange attractors in turbulence," vol. 898, pp. 366- 381. Springer-Verlag, 1981.

[23] B. de Vries and J. C. Principe, "The gamma model-a new neural model for temporal processing," Neural Networks, vol. 5, pp. 565-576, 1992.

[24] A. V. Oppenheim, R. W. Schafer, and J. R. Buck, Discrete-Time Signal Processing. Prentice-Hall, 1999.

[25] T. Lin, B. Horne, P. Tino, and C. Giels, "Learning longterm dependencies in narx recurrent neural networks," IEEE Trans. Neural Networks, vol. 7, no. 6, pp. 1329$1338,1996$.

[26] M. Youssef, A. Agrawala, and A. U. Shankar, "WLAN location determination via clustering and probability distributions," Pervasive Computing Commun., pp. 143150, 2003.

[27] A. Kushki, K. N. Plataniotis, and A. N. Venetsanopoulos, "Kernelbased positioning in wireless local area networks," IEEE Trans. Mobile Comput., vol. 6, no. 6, pp. 689-705, 2007.

[28] M. S. Grewal and A. P. Andrews, Kalman Filtering: Theory and Practice Using MATLAB. Wiley-IEEE Press, 2008. 